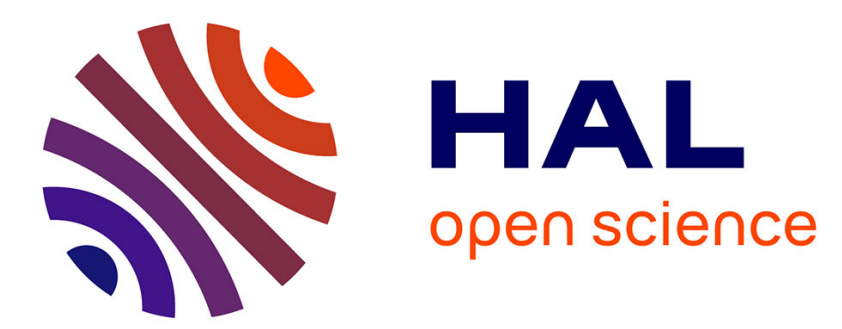

\title{
Sonographic appearance of a metastasis to the breast from a cerebellar medulloblastoma
}

Frederic Ternier

\section{To cite this version:}

Frederic Ternier. Sonographic appearance of a metastasis to the breast from a cerebellar medulloblastoma. Journal of Clinical Ultrasound, 2010, 10.1002/jcu.20698 . hal-00552406

\section{HAL Id: hal-00552406 \\ https://hal.science/hal-00552406}

Submitted on 6 Jan 2011

HAL is a multi-disciplinary open access archive for the deposit and dissemination of scientific research documents, whether they are published or not. The documents may come from teaching and research institutions in France or abroad, or from public or private research centers.
L'archive ouverte pluridisciplinaire HAL, est destinée au dépôt et à la diffusion de documents scientifiques de niveau recherche, publiés ou non, émanant des établissements d'enseignement et de recherche français ou étrangers, des laboratoires publics ou privés. 
Sonographic appearance of a metastasis to the breast from a cerebellar medulloblastoma

\begin{tabular}{|r|l|}
\hline Journal: & Journal of Clinical Ultrasound \\
\hline Manuscript ID: & JCU-09-222.R1 \\
\hline Wiley - Manuscript type: & Case Report \\
\hline Keywords: & medulloblastoma, metastasis, breast, sonography, core biopsy \\
\hline \multicolumn{2}{|l}{} \\
\hline
\end{tabular}

\section{S ScholaroNE"}


Medulloblastoma metastatic to breast diagnosed by corebiopsy

Sonographic appearance of a metastasis to the breast from a cerebellar medulloblastoma 
Keywords : Medulloblastoma, metastasis, breast, sonography, core biopsy

\begin{abstract}
We present the case of a 29 year old 29-year-old woman with a medulloblastoma of the cerebellum who developed a breast mass during the course of her disease. Core biopsy of the breast lesion revealed a metastatic medulloblastoma. Development of metastasis to the breast from medulloblastoma is very rare and the prognosis for such patients is poor. It is important to distinguish a primary breast cancer from metastasis to the breast, as both since the therapeutic options as well as the prognosis are very different. To our knowledge, it is the first report of the sonographic appearance of a medulloblastoma metastasic to the breast diagnosed by corebiopsy.
\end{abstract}




\section{Introduction}

Core-biopsy of the breast is now a well-established procedure for the evaluation of suspected breast mass before treatment ${ }^{1}$. We report a case of metastatic medulloblastoma to the breast which was diagnosed by core-biopsy under sonographic guidance. Metastatic tumors to the breast are rare and may mimic primary breast neoplasms. Preoperative diagnosis is extremely useful for determining appropriate treatment ${ }^{1,2}$. Medulloblastoma is a highly malignant tumor of the cerebellum that has a strong propensity to disseminate along the cerebrospinal pathways. Medulloblastoma is primarily a tumor seen in children and teenagers and which is particularly rare in adults. Standard treatment consists of complete excision of the tumor followed by radiation to the entire craniospinal axis with a boost to the primary tumor site. The role of adjuvant chemotherapy in adults is controversial. Relapse is most commonly within the posterior fossa and systemic metastases are rare. ${ }^{1}$

Extraneural metastases most commonly involve bone and bone marrow, followed by lymph nodes. ${ }^{1,2}$ Although even less common, metastases to the breast have been reported in adults. ${ }^{2-6}$ We report an extremely rare occurrence of metastatic medulloblastoma to the breast, describing its sonographic appearance and its diagnosis using ultrasound-guided core biopsy.

\section{Case report}

A 29 year old 29-year-old woman was referred to our institution for percutaneous biopsy of a palpable mass in her right breast. She had a history of médulloblastoma medulloblastoma of the cerebellum treated by complete surgical exeresis resection without the need for a shunt and radiotherapy ventricular shunt, followed by craniospinal radiotherapy, in another hospital, 5 years previously. Secondary Secondarily, 3 years after the excision of the médulloblastoma medulloblastoma, she developed a bicytopenia (Hemoglobin 7,4 g/dL, platelets $34 \times 10^{3} / \mathrm{L}$ ). A bone scan revealed diffuse osteoblastic desease disease. Bone marrow aspirate was positive for medulloblastoma cells. Nine cycles of carboplatin and etoposid were administred after which the patient was in complete remission. Two years after later, a tumor appeared in the upper outer quadrant of the right breast. Sonographic examination was performed using an a Xario scanner (Toshiba Inc, Tokyo, Japan) equiped with a 10-12 MHz a $14 \mathrm{MHz}$ linear- 
array transducer. The lesion was oval, hypoechoic, homogeneous, relatively wellcircumscribed with a vascular signal in power doppler sonography (Figure 1a, 1b). Sonographycally Sonographically guided core biopsy of the mass was performed using a automated biopsy gun (16-gauge) which allows obtaining a 20-mm- long core of tissue ) a $20 \mathrm{~mm}$-long sample of tissue to be taken (Achieve biopsy needle; Allegiance, Mc Graw park, IL, USA). Five pieces of good quality specimen were obtained for histologic study. Histopathology examination of the cores obtained showed a malignant tumor composed of small round cell synaptophysin positives synaptophysin-positive cells, confirming the diagnosis of medulloblastoma metastase metastasis (Figure 2a, 2b). Contrast enhanced computed tomography of the thorax, abdomen and pelvis revealed two homogeneous soft tissue masses in the mid and lower abdomen. The retroperitoneal lymph nodes were enlarged. She has now received 4 cycles of chemotherapy (ifosfamide and adriamycin) and had a good clinical response. Four cycles of chemotherapy (ifosfamide and adriamycin) were given, but the disease progressed over the ensuing months and the patient died 14 months after presenting with the breast mass.

\section{Discussion}

When a patient previously treated for a cancer presents with a breast lump, the differential diagnosis between benign and malignant (primary or secondary) breast tumor is based on clinical, imaging, and histologic criteria. It is important to make a distinction between these lesions since the optimal treatment is vastly different ${ }^{1,2,7,8,9}$.

The most important factor suggesting the diagnosis of secondary breast malignancy is a history of cancer. Nevertheless, metastases to the breast from extramammary neoplasms are rare and accounts for only $2 \%$ of all breast tumors. The incidence of breast metastasis ranges from $0.5 \%$ to $6.6 \%$ in autopsy series ${ }^{7,8 .}$ Apart from the lymphoproliferative diseases, the common primary malignancies which metastasize to the breast in decreasing order of frequency are are in order of decreasing frequency: melanoma, rhabdomyosarcoma, lung tumors, ovarian tumors, renal cell carcinoma, thyroid/cervical carcinomas and intestinal 
carcinoïd carcinoid $^{1,2,3,7}$. Metastases to the breast from a medulloblastoma is are rare. Médulloblastoma is primarily a tumour of the child and the teenager and is particularly rare in the adult, approximately $1 \%$ of the cerebral tumours of the adult. Medulloblastoma is the most common malignant brain tumor in children. In adults, however, medulloblastoma is a rare event and accounts for approximately $1 \%$ of all adult brain tumors ${ }^{1,10}$. It is an undifferenciated undifferentiated embryonal neuroepithelial tumor arising from the cerebellum with a tendency to infiltrate the subarachnoid space with subsequent spread via the cerebro-spinal fluid. Extraneural metastases are rare and have been reported in $10-30 \% 8-13 \%$ of cases ${ }^{4,1}$. The most frequently affected sites of dissemination are bone, bone marrow, lymph nodes, lungs, pleura, liver and others viscera. ${ }^{1,2}$ The mechanism of breast metastasis in medulloblastoma is not known. Both Lymphatic and haematogenous spread have both been suggested ${ }^{5,2}$.

Identifying secondary neoplasms of the breast clinically and by imaging is complex. The majority of metastases present as rapidly growing, painless, palpable, firm breast masses. Mammographic examination of these lesions usually reveals one or more well-circumscribed masses which are frequently interpreted as benign ${ }^{3,7-9}$. On sonography, the majority of metastases are round or oval, hypoechoic and circumscribed. The lesion may resemble a benign mass such as a fibroadenoma. Occasionally a variety of ultrasound findings have been reported including well or poorly defined hyper or hypoechoic solids masses with acoustic shadowing or increased through transmission ${ }^{1,2,7,8}$. In addition, vascularity of some lesions can be visualized with color-flow Doppler ${ }^{8}$. Previous report have proposed that the presence of intralesional vascularity is suggestive of a malignant masse ${ }^{6}$.

In our observation, clinic clinical and sonographic characteristics features of the breast lesion are aspecific. For problem solving, percutaneous biopsy (either fine needle or core biopsy) play a key role in the diagnosis of breast lesion ${ }^{1,7}$. Fine needle aspiration cytology (FNAB) is known for its high degree of accuracy, but results show considerable variability depending on the expertise of the cytopathologist. It was the preferred diagnostic procedure in the previous previously published reports of breast metastasis from médulloblastoma medulloblastoma $5,7,8,9,10,5,6$. Now, percutaneous large-core breast biopsy became has become a standard of care. Guided by sonography, core biopsy of breast lesion has a high accuracy. The 
advantage of performing a core biopsy is to obtain histologic samples in which the architectural description and tissue element recognition is are possible and which are superior to the cellular aspirate derived from $\mathrm{FNAB}^{1,7}$. Immunohistochemistry has an additional valuable role. Medulloblastomas are frequently positive for vimentin, synaptophysin and neuron-specific enolase (NSE) staining ${ }^{10,6}$.

The development of extraneural metastases of a medulloblastoma in breast tissue is associated with a poor prognosis. Nevertheless, as demonstrated by the current case, medulloblastoma is a chemosensitive tumor that is amenable to salvage treatment therapy. There have been several reports of prolonged remissions after salvage chemotherapy for extra-neural relapse $e^{4,10}$.

In conclusion, the occurrence of metastatic medulloblastoma to the breast is a possible event. Imaging has plays an important role in confirming the clinical suspicion of a lump, characterisation characterization of the lesion and guiding percutaneous biopsy. Overall survival of adult patients with recurrent medulloblastoma remains poor but meaningful extended survival can be obtained despite multiple recurrences. 


\section{References}

1. 7. Bartella L, Kaye J, Perry NM, et al. Metastases to the breast revisited : radiologicalhistopathological correlation. Clinical Radiology 2003;58:524-531.

1. Brandes AA, Franceschi E, Tosoni A, et al. Adult neuroectodermal tumors of posterior fossa (medulloblastoma) and of supratentorial sites (stPNET). Critical Reviews in Oncology/Hematology 2009;71:165-179.

2. Rochkind S, Blatt I, Sadeh M et al. Extracranial metastases of medulloblastoma in adults: literature review. J Neurol Neurosurg Psychiatry 1991;54:80-86.

2. Vizcaino I, Torregrossa A, Higueras V, et al. Metastasis to the breast from extramammary malignancies: a report of four cases and a review of literature. Eur. Radiol 2001;11:16591665.

3.9. McFarlane ME. Metastasis to the breast: A rare site for secondary malignancy[editorial]. International journal of surgery. 2006;4 : 204-205.

4.10 Wendland MM, Shrieve DC, Watson GA, et al. Extraneural metastatic medulloblastoma in an adult. Journal of neuro-oncology 2006; 78 :191-196.

5. Baliga M, Holmquist ND, Espinoza CG. Medulloblastoma metastatic to breast, diagnosed by fine-needle aspiration biopsy. Diagn Cytopathol 1994;10(1):33-36.

6. 8. Yang WT, Metreweli C. Sonography of nonmammary malignancies of the breast . AJR Am J Roentgenol 1999; Feb 172(2) : 343-348.

7.3. Brutschin P, Culver GJ. Extracranial metastases from medulloblastomas. Radiology 1973; 107 : 359-362. 
8.4 Brydon HL, Carey MP. Medulloblastoma metastasizing to a breast fibroadenoma: a case report. British Journal of Neurosurgery 1991; $5:$ 73-75.

9. Kapila K, Sarkar C, Verma K. Detection of metastatic medulloblastoma in a fine needle breast aspirate. Acta Cytolol 1996; 40(2) : 384-385.

10.6. Lamovec J, Pogaenik A. Metastatic medulloblastoma to the breast. Virchows Arch 2001; 439: 201-205.

\section{Illustrations}

Fig. 1a. Breast ultrasound. Sonogram shows an oval, relatively well-defined hypoechoic solid mass with no posterior shadowing.

Fig. 1a. Breast ultrasound. Transverse sonogram shows 19-mm (maximum diameter) oval, relatively well-defined hypoechoic solid mass with posterior acoustic enhancement.

Fig. 1b. Power Doppler examination shows evidence of significant intralesional vascularity.

Fig. 2a. Morphological aspect of the lesion in hematein eosin safran consistent with metastatic medulloblastoma, X 200 .

Fig. 2b. Intra-cytoplasmic immunnoreactivity for synaptophysin in numerous tumor cells, $\mathrm{X}$ 200. 
Sonographic appearance of a metastasis to the breast from a cerebellar medulloblastoma 
Keywords : Medulloblastoma, metastasis, breast, sonography, core biopsy

\begin{abstract}
We present the case of a 29-year-old woman with a medulloblastoma of the cerebellum who developed a breast mass during the course of her disease. Core biopsy of the breast lesion revealed a metastatic medulloblastoma. Development of metastasis to the breast from medulloblastoma is very rare and the prognosis for such patients is poor. It is important to distinguish primary breast cancer from metastasis to the breast, since the therapeutic options as well as the prognosis are very different.
\end{abstract}




\section{Introduction}

Medulloblastoma is a highly malignant tumor of the cerebellum that has a strong propensity to disseminate along the cerebrospinal pathways. Medulloblastoma is primarily a tumor seen in children and teenagers and which is particularly rare in adults. Standard treatment consists of complete excision of the tumor followed by radiation to the entire craniospinal axis with a boost to the primary tumor site. The role of adjuvant chemotherapy in adults is controversial. Relapse is most commonly within the posterior fossa and systemic metastases are rare. ${ }^{1}$ Extraneural metastases most commonly involve bone and bone marrow, followed by lymph nodes. ${ }^{1,2}$ Although even less common, metastases to the breast have been reported in adults. ${ }^{2-6}$ We report an extremely rare occurrence of metastatic medulloblastoma to the breast, describing its sonographic appearance and its diagnosis using ultrasound-guided core biopsy.

\section{Case report}

A 29-year-old woman was referred to our institution for percutaneous biopsy of a palpable mass in her right breast. She had a history of medulloblastoma of the cerebellum treated by complete surgical resection without the need for a ventricular shunt, followed by craniospinal radiotherapy, in another hospital 5 years previously. Secondarily, 3 years after the excision of the medulloblastoma, she developed a bicytopenia (Hemoglobin $7,4 \mathrm{~g} / \mathrm{dL}$, platelets $34 \times 10^{3}$ /L). A bone scan revealed diffuse osteoblastic disease. Bone marrow aspirate was positive for medulloblastoma cells. Nine cycles of carboplatin and etoposid were administred after which the patient was in complete remission. Two years later, a tumor appeared in the upper outer quadrant of the right breast. Sonographic examination was performed with a Xario scanner (Toshiba Inc, Tokyo, Japan) with a $14 \mathrm{MHz}$ linear-array transducer. The lesion was oval, hypoechoic, homogeneous, relatively well-circumscribed with a vascular signal in power doppler sonography (Figure 1a, 1b). Sonographically-guided core biopsy of the mass was performed using an automated biopsy gun (16-gauge) which allows a $20 \mathrm{~mm}$-long sample of tissue to be taken (Achieve biopsy needle; Allegiance, McGraw Park, IL, USA). Five pieces of good quality specimen were obtained for histologic study. Histopathology examination of the 
cores obtained showed a malignant tumor composed of small round synaptophysin-positive cells, confirming the diagnosis of medulloblastoma metastasis (Figure 2a, 2b). Contrastenhanced computed tomography of the thorax, abdomen and pelvis revealed two homogeneous soft tissue masses in the mid and lower abdomen. The retroperitoneal lymph nodes were enlarged. Four cycles of chemotherapy (ifosfamide and adriamycin) were given, but the disease progressed over the ensuing months and the patient died 14 months after presenting with the breast mass.

\section{Discussion}

When a patient previously treated for cancer presents with a breast lump, the differential diagnosis between benign and malignant (primary or secondary) breast tumor is based on clinical, imaging, and histologic criteria. It is important to make a distinction between these lesions since the optimal treatment is vastly different. ${ }^{7,8,9}$

Metastases to the breast from extramammary neoplasms are rare. The incidence of breast metastasis ranges from $0.5 \%$ to $6.6 \%$ in autopsy series. ${ }^{7,8}$ Apart from lymphoproliferative diseases, the common primary malignancies which metastasize to the breast are in order of decreasing frequency: melanoma, rhabdomyosarcoma, lung tumors, ovarian tumors, renal cell carcinoma, thyroid/cervical carcinomas and intestinal carcinoid. ${ }^{7}$ Metastases to the breast from a medulloblastoma are rare..$^{2-6}$ Medulloblastoma is the most common malignant brain tumor in children. In adults, however, medulloblastoma is a rare event and accounts for approximately $1 \%$ of all adult brain tumors ${ }^{1,10}$. It is an undifferentiated embryonal neuroepithelial tumor arising from the cerebellum, with a tendency to infiltrate the subarachnoid space with subsequent spread via the cerebro-spinal fluid. Extraneural metastases are rare and have been reported in $8-13 \%$ of cases ${ }^{1}$. The most frequently affected sites of dissemination are bone, bone marrow, lymph nodes, lungs, liver and other viscera. ${ }^{1,2}$ The mechanism of breast metastasis in medulloblastoma is not known. Both lymphatic and hematogenous spread have been suggested. ${ }^{2,5}$ 
The majority of metastases present as rapidly growing, painless, palpable, firm breast masses. Mammographic examination of these lesions usually reveals one or more wellcircumscribed masses which are frequently interpreted as benign. ${ }^{7-9}$ On sonography, the majority of metastases are round or oval, hypoechoic and circumscribed. The lesion may resemble a benign mass such as a fibroadenoma. Occasionally a variety of ultrasound findings have been reported, including well or poorly defined hyper or hypoechoic solid masses with acoustic shadowing or increased through transmission. ${ }^{7,8}$ In addition, vascularity of some lesions can be visualized with color-flow Doppler. ${ }^{8}$

In our observation, clinical and sonographic features of the breast lesion are aspecific. For problem solving, percutaneous biopsy (either fine-needle or core biopsy) plays a key role in the diagnosis of breast lesions. ${ }^{7}$ Fine-needle aspiration biopsy (FNAB) is known for its high degree of accuracy, but results show considerable variability depending on the expertise of the cytopathologist. It was the preferred diagnostic procedure in the previously published reports of breast metastasis from medulloblastoma. ${ }^{5,6}$ Now, percutaneous large-core breast biopsy has become a standard of care. Guided by sonography, core biopsy of breast lesions has high accuracy. The advantage of performing a core biopsy is to obtain histologic samples in which architectural description and tissue element recognition are possible and which are superior to the cellular aspirate derived from FNAB. ${ }^{7}$ Immunohistochemistry has an additional valuable role. Medulloblastomas are frequently positive for vimentin, synaptophysin and neuron-specific enolase (NSE) staining. ${ }^{6}$

The development of extraneural metastases of a medulloblastoma in breast tissue is associated with a poor prognosis. ${ }^{3,4,6}$ Nevertheless, as demonstrated by the current case, medulloblastoma is a chemosensitive tumor that is amenable to salvage therapy. There have been several reports of prolonged remissions after salvage chemotherapy for extra-neural relapse. ${ }^{10}$ In conclusion, the occurrence of metastatic medulloblastoma to the breast is a possible event. Imaging plays an important role in confirming the clinical suspicion of a lump, characterization of the lesion and guiding percutaneous biopsy. Overall survival of adult patients with recurrent medulloblastoma remains poor but meaningful extended survival can be obtained despite multiple recurrences. 


\section{References}

1. Brandes AA, Franceschi E, Tosoni A, et al. Adult neuroectodermal tumors of posterior fossa (medulloblastoma) and of supratentorial sites (stPNET). Critical Reviews in Oncology/Hematology 2009;71:165-179.

2. Rochkind S, Blatt I, Sadeh M, et al. Extracranial metastases of medulloblastoma in adults: literature review. J Neurol Neurosurg Psychiatry 1991;54:80-86.

3. Brutschin P, Culver GJ. Extracranial metastases from medulloblastomas. Radiology 1973;107:359-362.

4. Brydon HL, Carey MP. Medulloblastoma metastasizing to a breast fibroadenoma: a case report. British Journal of Neurosurgery 1991;5:73-75.

5. Baliga M, Holmquist ND, Espinoza CG. Medulloblastoma metastatic to breast, diagnosed by fine-needle aspiration biopsy. Diagn Cytopathol 1994;10:33-36.

6. Lamovec J, Pogaenik A. Metastatic medulloblastoma to the breast. Virchows Arch 2001; 439:201-205.

7. Bartella L, Kaye J, Perry NM, et al. Metastases to the breast revisited : radiologicalhistopathological correlation. Clinical Radiology 2003;58:524-531.

8. Yang WT, Metreweli C. Sonography of nonmammary malignancies of the breast. AJR Am J Roentgenol 1999; Feb 172:343-348.

9. McFarlane ME. Metastasis to the breast: A rare site for secondary malignancy [editorial]. International journal of surgery. 2006;4:204-205. 
10. Wendland MM, Shrieve DC, Watson GA, et al. Extraneural metastatic medulloblastoma in an adult. Journal of neuro-oncology 2006;78:191-196.

Fig. 1a. Breast ultrasound. Transverse sonogram shows 19-mm (maximum diameter) oval, relatively well-defined hypoechoic solid mass with posterior acoustic enhancement.

Fig. 1b. Power Doppler examination shows evidence of intralesional vascularity.

Fig. 2a. Morphological aspect of the lesion in hematein eosin safran consistent with metastatic medulloblastoma, X 200 .

Fig. 2b. Intra-cytoplasmic immunoreactivity for synaptophysin in numerous tumor cells, $X$ 200. 


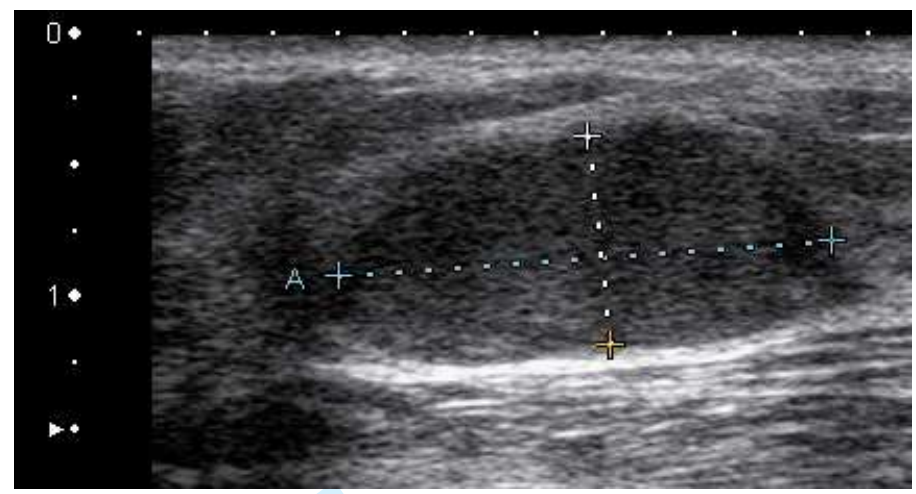

Fig. 1a. Breast ultrasound. Transverse sonogram shows 19-mm (maximum diameter) oval, relatively well-defined hypoechoic solid mass with posterior acoustic enhancement. 


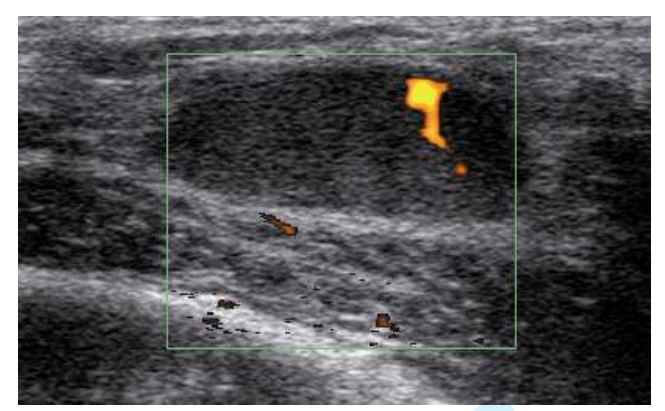

Fig. 1b. Power Doppler examination shows evidence of intralesional vascularity. 


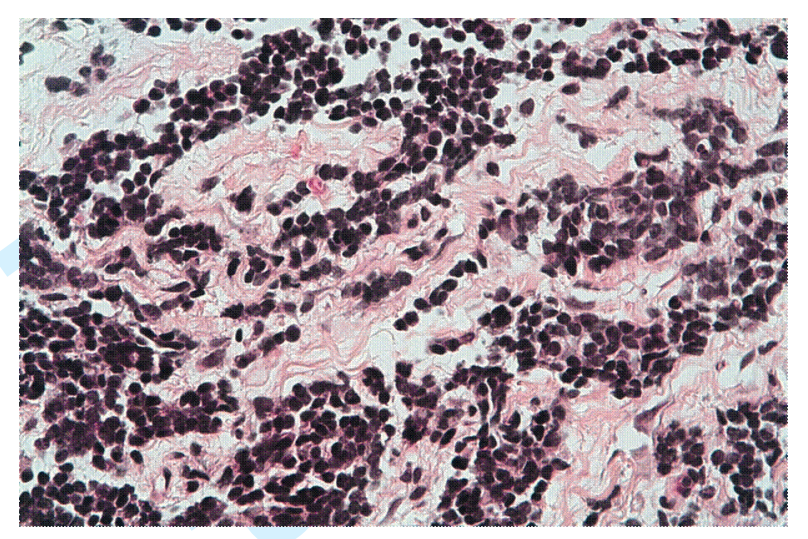

Fig. 2a. Morphological aspect of the lesion in hematein eosin safran consistent with metastatic medulloblastoma, $\times 200$. 


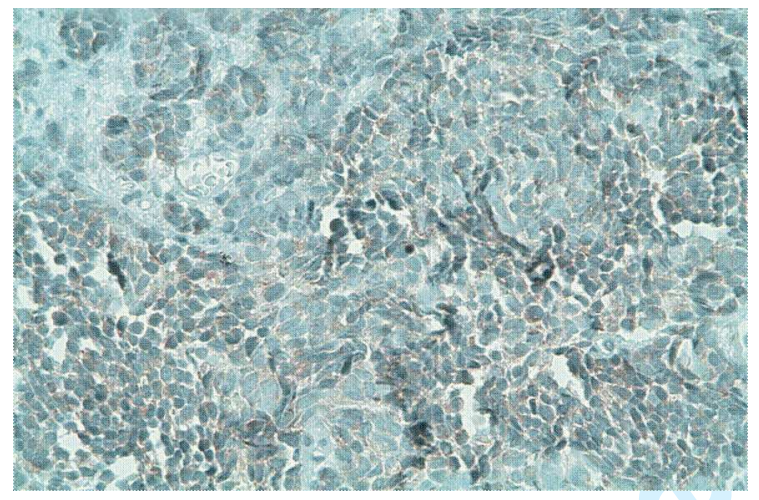

Fig. 2b. Intra-cytoplasmic immunoreactivity for synaptophysin in numerous tumor cells, X 200 